\title{
GLI2 inhibition reduces myofibroblast generation and kidney fibrosis
}

A new study provides experimental evidence that blocking the generation of myofibroblasts by inhibiting GLI2 - a transcriptional activator of hedgehog (Hh) signalling - might reduce fibrosis and slow the progression of kidney disease. Hh signalling regulates the fate of mesenchymal cells during kidney development and both the Hh pathway and GLI proteins have been implicated in the progression of organ fibrosis.

Benjamin Humphreys and colleagues previously showed that $\mathrm{GLI}^{+}$perivascular cells are a mesenchymal stem cell (MSC)like progenitor population that generates myofibroblasts after kidney injury. In their new study they investigated the functional role of $\mathrm{Hh}$ GLI signalling in these progenitors during fibrosis. "We believe that understanding how fibrosis occurs will lead to new treatments for patients with kidney disease," explains Humphreys. "The aim of our study was to provide proof-of-principle for this concept."

The researchers found that conditional knockout or inhibition of GLI2, but not GLI1, in cultured MSC-like progenitor cells induced cell-cycle arrest, whereas GLI2 overexpression activated $\mathrm{Hh}$ signalling and increased cell proliferation. In mice, GLI1 and GLI2 were specifically upregulated in cells of the myofibroblast lineage after kidney injury induced by unilateral ureteral obstruction. Conditional knockout of Gli2 in GLI1 ${ }^{+}$ cells or pharmacological inhibition of GLI2 induced myofibroblast cell-cycle arrest and reduced kidney fibrosis in this model. "Targeting GLI2 is an effective strategy to prevent myofibroblast proliferation and slow kidney disease progression in mice," says Humphreys.

To investigate the relevance of these findings to human disease, the

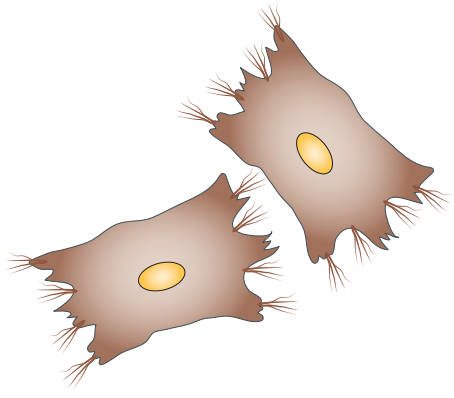

researchers analysed GLI1 and GLI2 mRNA expression and fibrosis severity in 10 tumour nephrectomy samples. They report that expression of GLI1 and GLI2 mRNA was higher in samples with high-grade fibrosis than in those with low-grade fibrosis and comment that these data suggest activation of the Hh-GLI pathway in human kidney fibrosis.

"Drugs that target GLI2 are already in clinical trials for diseases such as leukaemia and we might be able to repurpose them to slow kidney fibrosis in patients," says Humphreys. "However, it is critical to confirm that the pathway we identified in mice is active in humans before we think about designing a clinical trial of a Hh-GLI inhibitor."

Ellen F. Carney

Original article Kramann, R. et al. Pharmacological GL12 inhibition prevents myofibroblast cell-cycle progression and reduces kidney fibrosis. J. Clin. Invest. doi:10.1172/ JCl174929 